\title{
Control Predictivo en el espacio de estados de un captador solar tipo Fresnel
}

\author{
Antonio J. Gallego, Mario de la Rosa, Eduardo F. Camacho \\ Dpto. Ingeniería de Sistemas y Automática, Avenida de los descubrimientos s/nº 41092 (Sevilla) \\ agallego2@us.es, mardeltri@gmail.com, efcamacho@us.es
}

\section{Resumen}

Uno de los recursos más importantes para mejorar la eficiencia en los sistemas de energía solar, es el control avanzado. En general, con el uso de estrategias de control convencionales no se obtienen buenos desempeños en todo el rango de operación debido a la dinámica fuertemente no lineal de este tipo de sistemas, así como las múltiples fuentes de perturbaciones. En este trabajo se desarrolla un control predictivo basado en el espacio de estados para controlar un captador solar tipo Fresnel. Su comportamiento es analizado sobre el modelo de parámetros distribuidos no lineal de dicho captador.

Palabras clave: energía solar, colector Fresnel, control predictivo, observador LMI

\section{Introducción}

La necesidad de reducir el impacto de las fuentes de energía fósil tales como el carbón o el petróleo, produjo un gran interés en las fuentes de energía renovables a mediados de los 70. En los últimos 30 años, el uso de la energía solar ha experimentado un gran impulso, desde el punto de vista de la investigación y construcción de plantas comerciales. Una de las ventajas de la energía solar con respecto a otros tipos de energía renovables es la posibilidad de usar almacenamiento térmico [1].

Múltiples trabajos de investigación se llevaron a cabo en la planta experimental ACUREX en la plataforma Solar de Almería [2] y [3]. Desde el punto de vista de plantas comerciales, cabe citar las 13 plantas termosolares de tecnología cilindroparabólica (CCP) de Abengoa solar operativas en España (50 MW cada una) [4].

En general, el objetivo de control en plantas termosolares es mantener la temperatura de salida en torno a un punto de funcionamiento. Sin embargo, esto no es tarea sencilla debido a que hay múltiples fuentes de perturbaciones tales como la radiación solar, temperatura de entrada, eficiencia óptica, etc. La fuente de perturbación más importante es la radiación solar, cuya variación a lo largo de un día puede ser muy brusca [5].

Se han realizado múltiples pruebas de control en el campo del control de sistemas solares. Por ejemplo, en [6], se propone un control predictivo no lineal basado en un redes neuronales. En [7], se propone un MPC robusto basado en tubos para seguimiento de referencias. Estos dos controladores fueron probados en la planta experimental ACUREX. En [8], se propone un control predictivo combinado con un filtro de Kalman unscented para estimar estados y parámetros. En [9],se propone un método para hallar el punto óptimo de operación en plantas de tecnología CCP.

En este artículo, se propone un control predictivo en el espacio de estados el cual se aplica al captador solar tipo Fresnel ubicado en la Escuela Superior de Ingenieros de Sevilla. El diseño del controlador es similar al propuesto en [10]. El observador de estado elegido, es un observador de Luenberger debido a que, por su planteamiento de diseño, se pueden imponer restricciones de comportamiento y estabilidad, las cuales se pueden resolver mediante un LMI con restricciones politópicas en el plano discreto [11]. La ventaja de este esquema es que, la estrategia de control final puede plantearse como un problema lineal cuadrático $(\mathrm{QP})$ y resolverse de manera rápida y eficiente.

El artículo se organiza del siguiente modo: en la sección 2, se presenta el modelo matemático del captador. En la sección 3, se explica la estrategia de control. En la sección 4, se muestran algunos resultados de simulación y, en la sección 5 , se muestran las conclusiones.

\section{Descripción del modelo matemático del captador solar Fresnel}

El captador solar usado en este trabajo, pertenece a la planta de refrigeración solar ubicada en la terraza de la Escuela de Ingenieros de Sevilla. Esta está formada por el captador solar Fresnel, una máquina de absorción de doble efecto y un sistema de almacenamiento basado en materiales de cambio de fase [12] y [13]. 


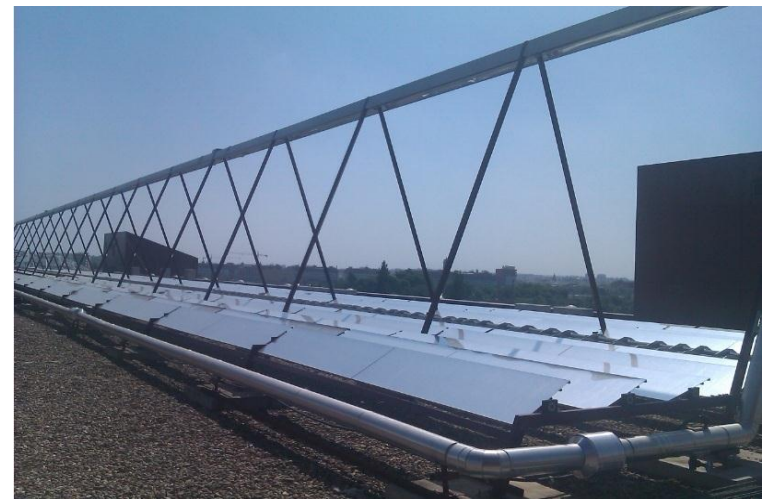

Figura 1: Campo de colectores Fresnel

El captador solar Fresnel está formado por 11 líneas de colectores Fresnel con una superficie total reflectante de $352 \mathrm{~m}^{2}$. Los colectores concentran la radiación solar en un tubo de metal de $64 \mathrm{~m}$ de longitud (Figura 1), calentando el agua a presión que circula por él y, de este modo, alimentando la máquina de absorción. Si hay más energía de la que la máquina de absorción puede usar, esta puede ser almacenada en el tanque de almacenamiento.

Con respecto al modelo matemático, se pueden usar dos enfoques: el modelo de parámetros concentrados y el modelo de parámetros distribuidos. El modelo de parámetros concentrados proporciona una descripción puntual del captador Fresnel [14]. Es una descripción más simple que el modelo de parámetros distribuidos.

La ventaja del modelo de parámetros distribuidos es que, además de considerar la distribución espacial de la temperatura del fluido, modela la transmisión de calor metal-fluido. En este trabajo se usa el modelo de parámetros distribuidos para realizar simulaciones y evaluar el desempeño del controlador propuesto. El modelo de parámetros distribuidos se puede describir con dos ecuaciones diferenciales en derivadas parciales [15] del siguiente modo:

$$
\begin{aligned}
\rho_{m} C_{m} S_{m} \frac{\partial T_{m}}{\partial t} & =I K_{o p t} n_{o} G-H_{l} G\left(T_{m}-T_{a}\right) \\
& -l_{p} H_{t}\left(T_{m}-T_{f}\right) \\
\rho_{f} C_{f} S_{f} \frac{\partial T_{f}}{\partial t}+ & \rho_{f} C_{f} q \frac{\partial T_{f}}{\partial l}=l_{p} H_{t}\left(T_{m}-T_{f}\right)
\end{aligned}
$$

Donde los subíndices $m$ y $f$ indican metal y fluido respectivamente. En el cuadro 1, se listan los parámetros del modelo y sus unidades.

La eficiencia óptica $K_{\text {opt }}$ está formada por factores como la reflectividad, absortancia del tubo, factor

\begin{tabular}{|c|l|r|}
\hline Símbolo & Descripción & Unidades \\
\hline $\mathrm{t}$ & Tiempo & $\mathrm{s}$ \\
$\mathrm{l}$ & Espacio & $\mathrm{m}$ \\
$\rho$ & Densidad & $\mathrm{kgm}^{-3}$ \\
$\mathrm{C}$ & Capacidad calorífica & $\mathrm{JK}^{-1} \mathrm{~kg}^{-1}$ \\
$\mathrm{~S}$ & Área de la sección & $\mathrm{m}^{2}$ \\
& transversal & \\
$T(x, y)$ & Temperatura & $\mathrm{K},{ }^{\circ} \mathrm{C}$ \\
$q(t)$ & Caudal & $\mathrm{m}^{3} \mathrm{~s}^{-1}$ \\
$I(t)$ & Radiación Solar & $\mathrm{Wm}^{-2}$ \\
$n_{o}$ & Eficiencia Geométrica & - \\
$K_{o p t}$ & Eficiencia óptica & - \\
$G$ & Apertura del colector & $\mathrm{m}$ \\
$T_{a}(t)$ & Temperatura ambiente & $\mathrm{K},{ }^{\circ} \mathrm{C}$ \\
$H_{l}$ & Coeficiente de pérdidas & $\mathrm{Wm}^{-2}{ }^{-1}$ \\
& térmicas & \\
$H_{t}$ & Coeficiente de transmi- & $\mathrm{Wm}^{-2}{ }^{\circ} \mathrm{C}^{-1}$ \\
& sión de calor metal- & \\
$l_{p}$ & fluido & Perímetro mojado \\
\hline
\end{tabular}

Cuadro 1: Descripción de los parámetros del modelo

de forma, etc. La eficiencia geométrica depende del ángulo de incidencia del sol y el factor de sombra [14].

La sistema de ecuaciones se resuelve diviendo el tubo en 64 segmentos de $1 \mathrm{~m}$ de longitud y un tiempo de integración de $0.5 \mathrm{~s}$.

El coeficiente de transmisión de calor $H_{t}$ depende tanto de la temperatura como del caudal [16]. El valor de los otros parámetros se puede hallar en [17].

\section{MPC en el espacio de estados}

En esta sección se describe el MPC implementado en este trabajo. Las matrices para el modelo en el espacio de estados se obtienen a partir de las ecuaciones del modelo de parámetros distribuidos (ecuación (1)). Con el objetivo de reducir la carga computacional del controlador, el modelo lineal se obtiene dividiendo el tubo en 4 segmentos en lugar de los 64 requeridos en el modelo completo.

El objetivo de control de un sistema de energía solar como el captador Fresnel, es mantener la temperatura de salida en torno a una referencia deseada, usando para tal fin, el caudal como variable manipulable.

Debido a que solo las temperaturas de entrada y salida son accesibles, el resto de temperaturas deben ser estimadas. Para ello, se ha diseñado un observador de estado cuya ganancia es calculada por medio de un problema de asignación de polos 
robusto, cuya solución puede ser obtenida mediante LMIs. Se considera incertidumbre politópica en la dinámica de la planta [11].

En primer lugar, se presenta el procedimiento de obtención de las matrices del modelo lineal y, posteriormente, se describe el método para computar la ganancia del observador de Luenberger.

\subsection{Obtención de las matrices del modelo lineal}

En esta subsección se describe el procedimiento de obtención de las matrices lineales. El modelo lineal del sistema de ecuaciones diferenciales (ecuaciones (1)), está formado por un conjunto de matrices que dependen de las entradas y los estados del sistema. Sea $x$ el vector de estados formado por las temperaturas de los 4 segmentos del metal y del fluido, $T_{i n}$ es la temperatura de entrada, $q$ el caudal de agua, $I_{e}=I K_{o p t} n_{o}$ es la radiación solar efectiva y $T_{a}$ es la temperatura ambiente.

El modelo lineal, en tiempo contínuo se puede computar usando (2):

$$
\begin{array}{rl}
\dot{x}(t)=A x(t) & +B u(t)+B_{d} d(t) \\
y(t) & =C x(t) \\
u(t)=q & d(t)=\left[\begin{array}{lll}
T_{\text {in }} & I_{e} & T_{a}
\end{array}\right]^{\top}
\end{array}
$$

Las matrices lineales se puede obtener del siguiente modo:

$$
\begin{aligned}
& P_{0}=\frac{-H_{l} G-l_{p} H_{t}}{\rho_{m} C_{m} S_{m}} \quad P_{1}=\frac{l_{p} H_{t}}{\rho_{m} C_{m} S_{m}} \\
& P_{2}=\frac{l_{p} H_{t}}{\rho_{f} C_{f} S_{f}} \quad P_{3}=\frac{q}{S_{f} \Delta l} \\
& P_{4}=-P_{2}-P_{3} \\
& A=\left(\begin{array}{cccccccc}
P_{0} & 0 & 0 & 0 & P_{1} & 0 & 0 & 0 \\
0 & P_{0} & 0 & 0 & 0 & P_{1} & 0 & 0 \\
\ldots & \ldots & \ldots & \ldots & \ldots & \ldots & \ldots & \ldots \\
0 & 0 & 0 & P_{0} & 0 & 0 & 0 & P_{1} \\
P_{2} & 0 & 0 & 0 & P_{3} & 0 & 0 & 0 \\
0 & P_{2} & 0 & 0 & P_{4} & P_{3} & 0 & 0 \\
\ldots & \ldots & \ldots & \ldots & \ldots & \ldots & \ldots & \ldots \\
0 & 0 & 0 & P_{2} & 0 & 0 & P_{4} & P_{3}
\end{array}\right) \\
& B=\left[\begin{array}{llll}
0_{1 x 4} & \frac{1}{S_{f} \Delta l} & \cdots & \frac{1}{S_{f} \Delta l}
\end{array}\right]^{\top} \\
& B_{\text {Tin }}=\left[\begin{array}{lll}
0_{1 x 4} & \frac{q}{S_{f} \Delta l} & 0_{1 x 3}
\end{array}\right]^{\top} \\
& B_{I e}=\left[\begin{array}{ll}
1_{1 x 4} \cdot \frac{G}{\rho_{m} C_{m} S_{m}} & 0_{1 x 4}
\end{array}\right]^{\top} \\
& B_{T a}=\left[\begin{array}{llll}
\frac{H_{l} G}{\rho_{m} C_{m} S_{m}} & \cdots & \frac{H_{l} G}{\rho_{m} C_{m} S_{m}} & 0_{4 x 1}
\end{array}\right]^{\top}
\end{aligned}
$$

$$
\begin{gathered}
B_{d}=\left[\begin{array}{llll}
B_{T i n} & B_{I e} & B_{T a}
\end{array}\right]^{\top} \\
C=\left[\begin{array}{llllllll}
0 & 0 & 0 & 0 & 0 & 0 & 0 & 1
\end{array}\right]
\end{gathered}
$$

Es importante resaltar que $A$ y $B_{T a}$ depende de los estados del sistema y de sus parámetros; $B_{\text {Tin }}$ es función del caudal de agua $u$. Como ha sido comentado en la sección 2 , los parámetros $H_{l}, p_{f}$, $C_{f}$ y $H_{t}$ dependen de la temperatura y del caudal de agua, mientras que $\rho_{m}, C_{m}, S_{f}, S_{m}$ y $G$ son constantes. El modelo lineal se discretiza con un tiempo de muestreo de $20 \mathrm{~s}$. A partir de ahora, nos referiremos a las matrices discretas cuando hablemos de $A, B$ y $B_{d}$.

\subsection{Formulación del MPC en el espacio de estados}

Desde los años 80, el control predictivo basado en modelo (MPC), ha experimentado un importante impulso en el campo de la investigación y de las aplicaciones industriales tales como plantas químicas y refinerías de petróleo.

Básicamente, la estrategia de un esquema de control MPC consiste en los siguientes 3 pasos $[18],[19]$ :

- El uso de un modelo matemátcio para predecir la evolución futura del proceso en un intervalo de tiempo (horizonte).

- Calcular una secuencia de acciones de control que minimizan una función objetivo.

- Aplicar solo la primera acción calculada (horizonte deslizante), y recalcular la secuencia en cada periodo de muestreo.

La principal diferencia en las diferentes estrategias de control predictivo, es la tipología del modelo: lineal o no lineal. Si el modelo es lineal, el problema de optimización asociado puede ser resuelto eficientemente y se puede alcanzar el óptimo global [20]. Si el modelo es no lineal, el problema de optimización requiere la resolución de un problema de programación no lineal, el cual es computacionalmente más costoso de resolver y alcanzar el óptimo global no está asegurado. Particularmente en un proceso como el sistema de energía solar de este artículo, para poder resolver el problema no lineal en el tiempo de muestreo requerido, es necesario elegir horizontes pequeños [15].

Por otra parte, el uso de estrategias de control lineal en una planta donde la dinámica es muy no lineal, tiene algunos inconvenientes. El desempeño 
de los controladores lineales se deteriora si el sistema evoluciona lejos del punto de funcionamiento de diseño. En los sistemas de energía solar, la dinámica de la planta deviene muy lenta con grandes retardos a bajos caudales [21]. Para tratar de solucionar este problema, en este artículo, las matrices del modelo de predicción del MPC cambian con el punto de funcionamiento.

Ante la dificultad de predecir la evolución de algunas entradas del sistema tales como la temperatura de entrada $T_{i n}$, y la radiación efectiva $I_{e} \mathrm{y}$ la temperatura ambiente $T_{a}$, la evolución de estas variables a lo largo del horizonte de predicción se consideran constantes.

Dado que la variable manipulable es el caudal, dos restricciones son tenidas en cuenta:

- caudal de agua entre 2 y $13 \mathrm{~m}^{3} / \mathrm{h}$.

- variación máxima de la señal de control en cada periodo de muestreo de $\pm 2 \mathrm{~m}^{3} / \mathrm{h}$.

La bomba de agua se modela como un sistema de primer orden con una ganancia unitaria y una constante de tiempo de 6 segundos. El controlador MPC requiere la solución de un problema QP, resuelto con el toolbox de optimización de Matlab.

$$
\begin{gathered}
\operatorname{mín}_{\Delta u} J= \\
\sum_{k=0}^{N_{y}}\left(y(t+k \mid t)-y_{r e f}(t+k)\right)^{T} Q\left(y(t+k \mid t)-y_{r e f}(t+k)\right) \\
+\sum_{k=0}^{N_{c}} \Delta u^{T}(t+k) R \Delta u(t+k)
\end{gathered}
$$

s.t:

$$
\begin{gathered}
\Delta u_{\min } \leq \Delta u(t+k) \leq \Delta u_{\max }, k=1, \ldots, N_{c} \\
u_{\text {min }} \leq u(t+k \mid t) \leq u_{\max }, k=1, \ldots, N_{c} \\
u(t+k \mid t)=u(t+k-1)+\Delta u(t+k-1), k=1, \ldots, N_{c} \\
x(t+k \mid t)=A x(t+k-1 \mid t)+B u(t+k-1 \mid t)+B_{d} d(t) \\
y(t+k)=C x(t+k), k=1, \ldots, N_{y}
\end{gathered}
$$

$N_{y}$ y $N_{c}$ son el horizonte de predicción y de control respectivamente.

\subsection{Obtención de la ganancia de observación $L$}

Tal como se ha mencionado anteriormente, solo algunas variables son directamente medibles. El resto de estados deben ser estimados mediante un observador. El observador elegido en este trabajo es un observador de Luenberger, cuya ganancia es calculada mediante una ubicación de polos robusta. Este enfoque permite que el problema pueda ser planteado como un conjunto de LMIs y resuelto mediante un toolbox de resolución de LMIs.

Para el diseño de este observador se imponen ciertos requisitos de desempeño en todo el rango de operación de la planta. Para cubrir todo el rango de operación, se ha empleado un politopo de 4 vértices (LDI) que representa el comportamiento en el rango de variación esperado en la temperatura y en el agua, variables de las cuales depende la dinámica del sistema. Las ecuaciones que describen la dinámica del observador de estado son:

$$
\begin{gathered}
\hat{x}(k+1)=A(k, x, u) \hat{x}(k)+B u(k)+B_{d} d(k)+ \\
L(\hat{y}(k)-y(k)) \\
\hat{y}(k+1)=C \hat{x}(k)
\end{gathered}
$$

Donde $A(k, x, u) \in \mathbf{C o}\left\{A_{1}, \ldots, A_{4}\right\}$.

El error de estimación se define como $e(k)=$ $\hat{x}(k)-x(k)$ y su dinámica viene dada por la siguiente ecuación:

$$
e(k+1)=(A(k, x, u)+L C) e(k)
$$

El objetivo del problema LMI que se formula en este artículo, es hallar una ganancia de observación $L$ de modo que $e(k)$ converja a 0 para $k \rightarrow \infty$ para el sistema descrito por el politopo LDI. Los requerimientos de diseño se imponen mediante restricciones en la ubicación de polos, es decir, no solo los polos deben estar dentro del círculo unidad (estabilidad), si no que se impone que su ubicación para los vértices del politopo, esté dentro de un radio máximo. Es interesante hacer notar que, para evitar términos no lineales en el LMI, se formula el problema con $(A(k, x, u))+L C)^{\top}$, dado que los autovalores de una matriz cuadrada, son lo mismos que los de su traspuesta.

Esto, matemáticamente, se expresa del siguiente modo: hallar una matriz $W$ de tal modo que los siguientes LMIs se satisfacen para cada vértice del politopo [22]:

$$
\begin{gathered}
\min _{W, Y} r \\
A_{i}^{\top} W+C^{\top} Y+W A+Y^{\top} C>0
\end{gathered}
$$




$$
\left[\begin{array}{cc}
-r W & A^{\top} W+C^{\top} Y \\
W A+Y^{\top} C & -r W
\end{array}\right]<0
$$

Con $Y=L^{\top} W$ y $r \in(0,1)$, corresponden a las siguientes condiciones para la ubicación de polos [11], [23]:

- $\operatorname{Re}\left(\lambda\left(A_{i}+L C\right)\right)>0$

- $\left|\lambda\left(A_{i}+L C\right)\right|<r$

Aunque las condiciones impuestas en (14b) son no lineales, realmente son condiciones bilineales, con lo que el problema se puede relajar iterativamente, buscando el valor mínimo de $r$ para el cual el problema LMI mantiene la factibilidad. La matriz de observación obtenida viene dada por:

$$
\left.L=\begin{array}{lllc}
-0,1469 & -0,6427 & -1,1269 & -0,9801 \\
-0,1376 & -0,6004 & -1,0615 & -0,9618
\end{array}\right]^{T}
$$

En la figura 2, se muestran los polos para los vértices del LDI, así como el mínimo valor de $r$ obtenido.

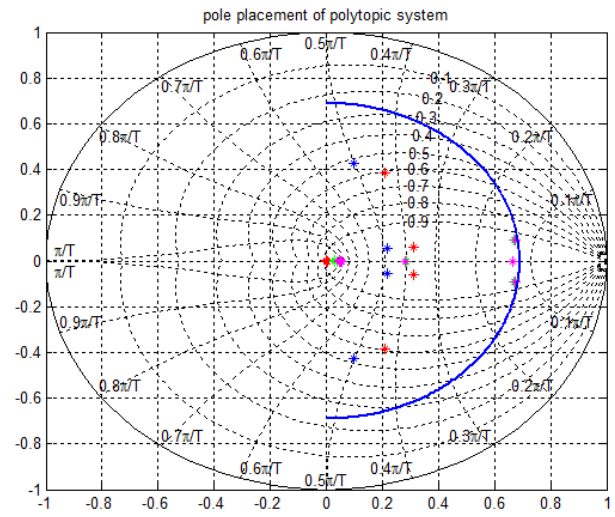

Figura 2: Resultado de la ubicación de polos obtenida resolviendo los LMIs propuestos

\subsection{MPC con estimador de perturbaciones}

Uno de los problemas que tiene el control predictivo en el espacio de estados es la aparición de un error en régimen permanente si el modelo no es suficientemente exacto. Para paliar este problema, el método propuesto en [24] se usa para compensar las diferencias entre el modelo lineal y la planta. Si consideramos un modelo lineal en el espacio de estados de la forma:

$$
\begin{gathered}
x_{k+1}=A x_{k}+B u(k)+B_{d} d(k) \\
y_{k}=C x_{k}
\end{gathered}
$$

$$
u_{k}=u_{k-1}+\Delta u_{k}
$$

La clave es estimar una señal de control virtual $\hat{u}_{k}$ que no es necesariamente igual a la señal de control real $u_{k}$ aplicada a la planta. Cualquier diferencia entre la planta y el modelo es incluida en esta señal virtual, que actúa como integrador de perturbaciones. La formulación final del observador de estados vienen dada por (17):

$$
\begin{gathered}
\hat{x}(k+1)=A \hat{x}(k)+B(\hat{u}(k)+\Delta u(k))+B_{d} d(k) \\
+L(\hat{y}(k)-y(k)) \\
\hat{u}(k+1)=\hat{u}(k)+\Delta u(k)+L_{u}(y(k)-\hat{y}(k))
\end{gathered}
$$

\subsection{Esquema final de control}

En esta subsección se muestra el esquema de control final. Cada 20 segundos, el sistema de adquisición de datos lee los sensores y el observador de estado calcula los estados estimados. Esta estimación se pasa al bloque de control MPC, que computa las matrices lineales y resuelve el problema de optimización (11). El resultado es la señal $q_{\text {pred }}$, la cual es añadida al controlador feedforward $q_{f f}$. La suma de ambas es la señal de control aplicada a la planta.

La razón de incluir un controlador feedforward es que ayuda al rechazo de perturbaciones [25]. El feedforward se calcula usando el modelo de parámetros concentrados como sigue [14]:

$$
q_{f f}=\frac{I K_{o p t} n_{o} S-H_{l} S\left(\left(T_{r e f}+T_{i n}\right) / 2-T_{a}\right)}{P_{c p}\left(y_{r e f}-T_{i n}\right)}
$$

Donde $y_{r e f}$ es la referencia de temperatura, $S$ es la superficie reflectante de $352 \mathrm{~m}^{2}$ y $P_{c p}$ es un término que incluye la entalpía del fluido y otros factores geométricos [15]. La figura 3, muestra el esquema de control final:

\section{Resultados}

Se han realizado dos simulaciones comparando el comportamiento del MPC propuesto con un controlador PI+feedforward. El PI ha sido diseñado usando multiples simulaciones con diferentes condiciones de radiación y caudal de agua, y sus ganancias se han obtenido mediante un proceso de optimización offline, para asegurar que el comportamiento es adecuado en todo el rango de funcionamiento, evitando inestabilidades. Su expresión viene dada por la ecuación (19)

$$
u_{P I}(k)=-0,2546 e(k)-0,003122 \Sigma_{e}(k)
$$




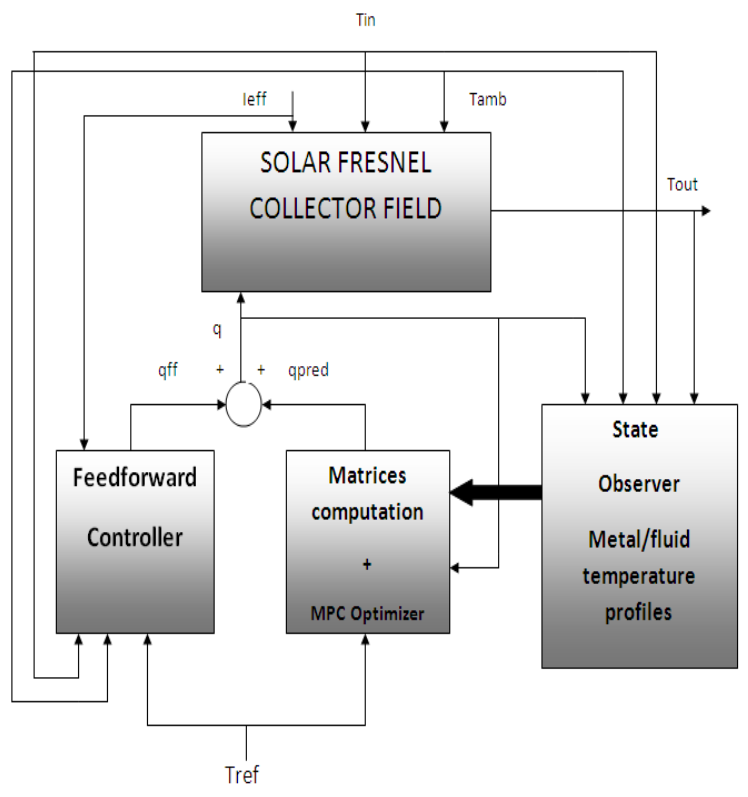

Figura 3: Esquema de control final

Donde $e(k)$ y $\Sigma_{e}(k)$ son el error y su integral discreta respectivamente. Para el controlador MPC se han elegido los siguientes parámetros: $N_{y}=15$, $N_{c}=10, Q=I$ y $R=120$.

La figura 4 muestra una simulación de un día claro donde el set-point cambia a lo largo de todo el test. El valor inicial de $T_{\text {in }}$ y $T_{\text {out }}$ es $90{ }^{\circ} \mathrm{C}$ y 99 ${ }^{\circ} \mathrm{C}$ respectivamente. Como se puede comprobar, el desempeño del PI es similar al del MPC para caudales medios-altos, pero para bajos caudales, el desempeño del PI se deteriora produciendo comportamiento oscilatorio, mientras que el MPC logra desempeños buenos en todo el rango.

En la figura 5 se simula un día con perturbaciones en la temperatura de entrada y en la radiación solar. La temperatura de entrada y la temperatura de salida tienen un valor inicial de $152{ }^{\circ} \mathrm{C}$ y 159 ${ }^{\circ} \mathrm{C}$ respectivamente. A las $12.4 \mathrm{~h}$ la temperatura de entrada incrementa su valor hasta $\operatorname{los} 156^{\circ} \mathrm{C}$, que produce un decremento en la temperatura de salida debido a la acción del feedforward, pero ambos controladores recuperan el seguimiento del set-point. A las $13.25 \mathrm{~h}$, debido a nubes dispersas, la radiación cae a 0 y el caudal de agua se viene al mínimo de $2 \mathrm{~m}^{3} / \mathrm{h}$, para minimizar la caída de temperatura. Aun así, en estas condiciones no es posible mantener la referencia de temperatura. Una vez que la nube ha pasado, el MPC recupera el seguimiento de set-point, de manera más suave. Desde $13.8 \mathrm{~h}$ hasta las $14.1 \mathrm{~h}$, una segunda nube cubre el campo de colectores y la temperatura de entrada disminuye produciendo otra saturación en la acción de control. La parte final del test consiste en una serie de escalones incrementales en el set-point. En este punto se puede ver que el PI deteriora su comportamiento en comparación con el MPC. Como se esperaba el desempeño y la robustez del MPC son superiores.

Con respecto al observador de estado, la figura 6 muestra la estimación de los perfiles del metal y del fluido. Como puede verse, los perfiles estimados están cercanos (con las lógicas diferencias) a los valores obtenidos con el modelo no lineal de parámetros distribuidos. El estimador de estado muestra convergencia rápida a pesar de las perturbaciones y cambios de referencia.
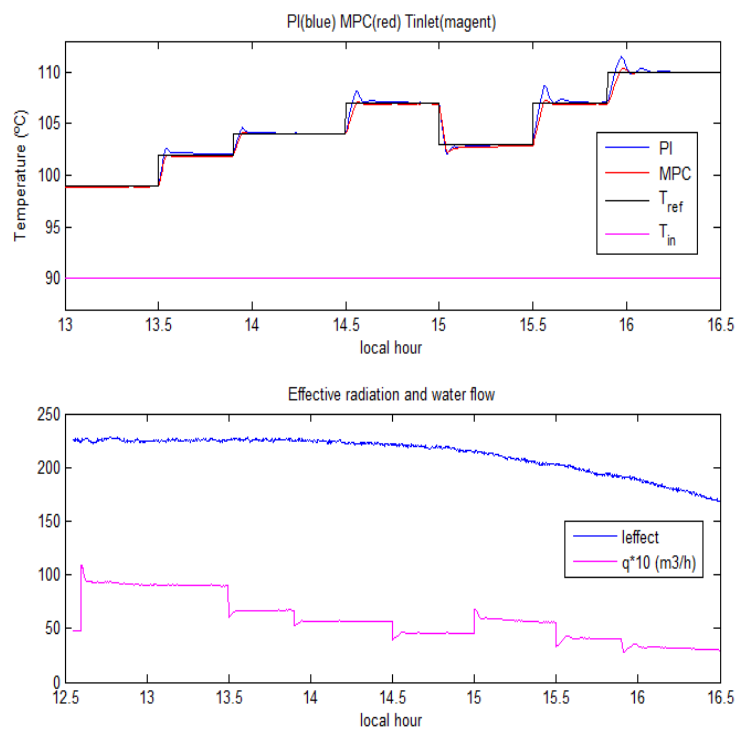

Figura 4: Día claro a baja temperatura. Comparación entre PI y MPC
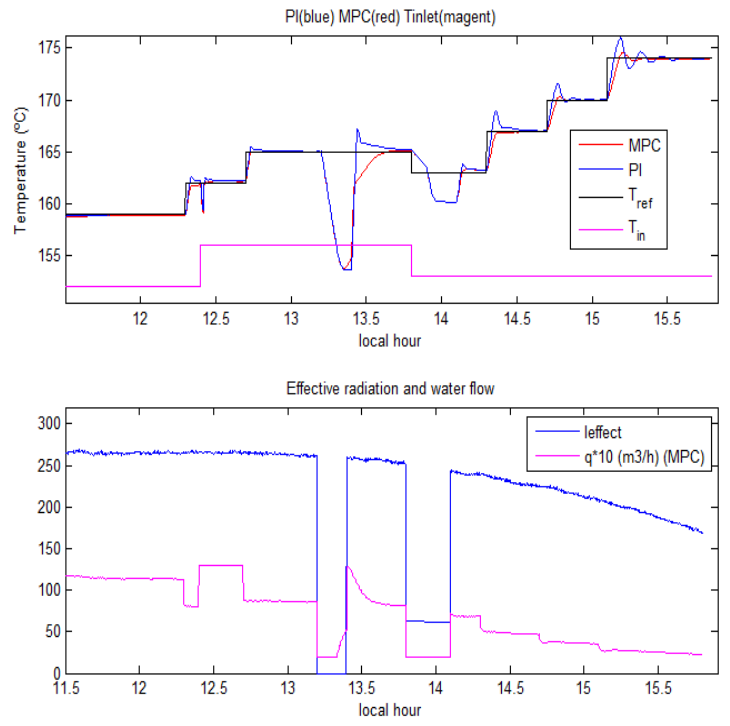

Figura 5: Día claro a alta temperatura. Comparación entre PI y MPC 

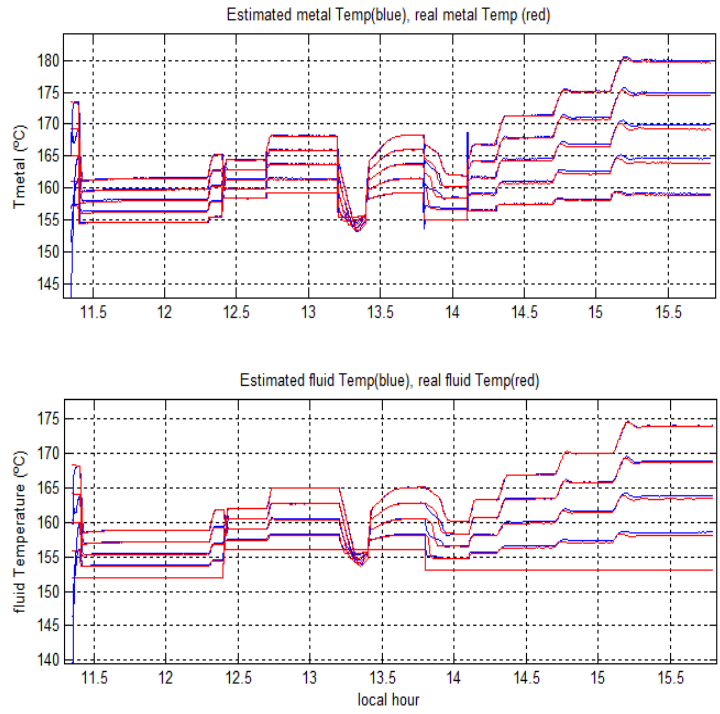

Figura 6: Día claro a alta temperatura: Desempñeo del observador de estado

\section{Conclusiones}

En general, el uso de estrategias de control lineales no es suficiente para garantizar un comportamiento eficiente en todo el rango de operación de las plantas solares. En este artículo, se ha diseñado un MPC basado en observador de estado aplicado a una planta de colectores Fresnel.

El observador de estado ha sido obtenido mediante el planteamiento de varios LMIs, imponiendo restricciones en dinámica y estabilidad. El desempeño del MPC se ha comparado con un PI, cuyos parámetros han sido obtenidos mediante optimización para diferentes condiciones. El desempeño del MPC se ha mostrado superior en las pruebas realizadas.

El observador de estado ha mostrado también rapidez de convergencia y buena estimación en los estados.

\section{Agradecimientos}

Los autores quieren agradecer a la junta de Andalucía y al Ministerio de Educación por financiar el trabajo mediante los proyectos Gestión Óptima de Edificios de Energía Cero (P11-TEP8129) y Control Predictivo de Microrredes Reconfigurables con Almacenamiento Híbrido y Móvil (DPI2016-78338-R).

\section{Referencias}

[1] E.F. Camacho, M. Berenguel, F.R. Rubio, and D. Martínez. Control of Solar Energy Systems. Springer-Verlag, 2012.

[2] E.F. Camacho, F.R. Rubio, M. Berenguel, and L. Valenzuela. A survey on control schemes for distributed solar collector fields. part I: Modeling and basic control approaches. Solar Energy, 81:1240-1251, 2007.

[3] E.F. Camacho, F.R. Rubio, M.Berenguel, and L.Valenzuela. A survey on control schemes for distributed solar collector fields. part II: Advanced control approaches. Solar Energy, $2007 \mathrm{~b}$

[4] Abengoa Solar. Abengoa solar: Energía solar para un mundo sostenible, 2012.

[5] A. J. Gallego and E. F. Camacho. Adaptative state-space model predictive control of a parabolic-trough field. Control Engineering Practice, 20(9):904-911, 2012.

[6] P.Gil, J.Henriques, P.Carvalho, H.Duarte Ramos, and A.Dourado. Constrained adaptative non-linear neural model-based predictive control of a distributed collector field. Proc. 2nd IHP Workshop, 2002.

[7] D.Limon, I.Alvarado, T.Alamo, M.Ruíz, and E.F Camacho. Robust control of the distributed solar collector field acurex using MPC for tracking. Proceedings of the 17th World Congress The International Federation of Automatic Control, pages 958-963, 2008.

[8] G.Pin, M.Falchetta, and G.Fenu. Modeling and control of concentrating solar power systems: a discrete-time adaptative scheme for temperature control in molten-salt solar collector-fields. In Solar Collectors: Energy Conservation, Design and Applications, Series: Renewable Energy: Research, Development and Policies. Nova Publishers:15-39, 2009.

[9] E. F. Camacho and A. J. Gallego. Optimal operation in solar trough plants: a case study. Solar Energy, 95:106-117, 2013.

[10] A. J. Gallego, F. Fele, E. F. Camacho, and L. J. Yebra. Observer-based model predictive control of a solar trough plant. Solar Energy, 97:426-435, 2013.

[11] Mahmoud Chilali and Pascal Gahinet. $\mathrm{H}_{\infty}$ design with pole placement constraints: An LMI approach. IEEE TRANSACTIONS ON AUTOMATIC CONTROL, 41:358-367, March 1996.

[12] Pablo Bermejo, Francisco Javier Pino, and Felipe Rosa. Solar absorption cooling plant in seville. Solar Energy, 84:1503-1512, 2010.

[13] A. J. Gallego, A. Ruíz-Pardo, A. CerezuelaParish, , J. Sánchez Ramos, C. MartínMacareno, L. F. CabezaCabeza, E. F. Camacho, and E. Oró. Mathematical modeling of 
a pcm storage tank in a solar cooling plant. Solar Energy, 93:1-10, 2013.

[14] María Robledo, Juan M. Escaño, Amparo Núnez, Carlos Bordons, and Eduardo F. Camacho. Development and experimental validation of a dynamic model for a fresnel solar collector. 18th IFAC World Congress., October 2010 .

[15] E.F Camacho, F.R Rubio, and M.Berenguel. Advanced control of solar plants. 1997.

[16] Frank Kreith, Raj M. Manglik, and Mark S. Bohn. Principles of heat transfer. Cengage Learning, seventh edition, 2011.

[17] Antonio J. Gallego. Control Predictivo de sistemas de energía solar distribuidos. $\mathrm{PhD}$ thesis, Universidad de Sevilla, June 2014.

[18] E.F. Camacho and C. Bordons. Model Predictive Control $2^{\circ}$ Ed. Springer Verlag, 2004.

[19] J.B Rawlings and D.Q Mayne. Model Predictive Control: Theory and Design. Cheryl M. Rawlings, 2009.

[20] D.Q Mayne, J.B Rawlings, C.V Rao, and P.O.M Scokaert. Constrained model predictive control: Stability and optimality. Automatica, 36:789-814, 2000.

[21] Ricardo Carmona. Análisis, Modelado y control de un campo de colectores solares distribuidos con sistema de seguimiento en un eje. PhD thesis, Universidad de Sevilla, 1985.

[22] Stephen Boyd, Laurent El Ghaoui, Eric Feron, and Venkataramanan Balakrishnan. $\mathrm{Li}_{-}$ near Matrix Inequalities in System and Control Theory. Society for Industrial and Applied Mathematics, 1994.

[23] J.M Gomes da Silva and S.Tarbouriech. Local stabilization of discrete-time linear systems with saturating controls: An lmi-based approach. IEEE TRANSACTIONS ON AUTOMATIC CONTROL, 46:119-125, January 2001.

[24] Urban Maedera, Francesco Borrelli, and Manfred Morari. Linear offset-free model predictive control. Automatica, 45:2214-2222, 2009.

[25] E.F. Camacho, F.R. Rubio, and F.M. Hughes. Self-tuning control of a solar power plantwith a distributed collector field. IEEE Control Systems, 0272- 1708/92/:7278, 1992. 